Preprint

UCRL-CONF-203930

\title{
Roughness Scaling of Fracture Surfaces in Polycrystalline Materials
}

Seppälä E. T., Reed B. W., Kumar M., Minich R. W., Rudd R. E.

Materials Research Society Spring Meeting 2004, San Francisco,

Lawrence

Livermore CA, April 12 - 16, 2004

National

Laboratory

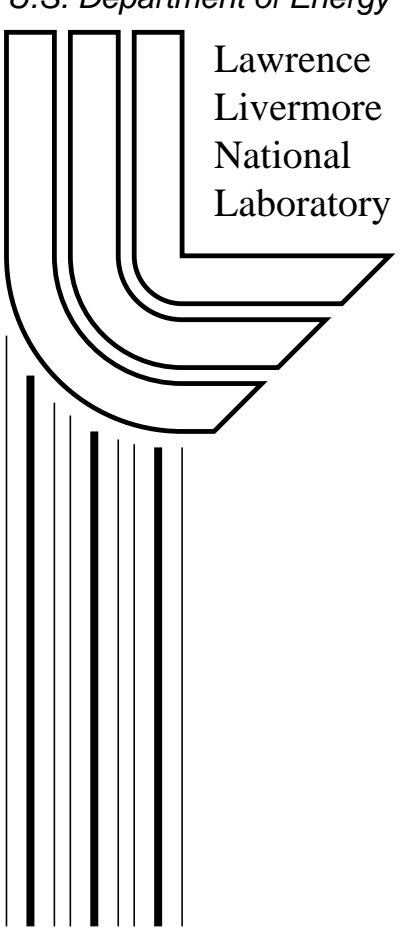

May 4, 2004 


\section{DISCLAIMER}

This document was prepared as an account of work sponsored by an agency of the United States Government. Neither the United States Government nor the University of California nor any of their employees, makes any warranty, express or implied, or assumes any legal liability or responsibility for the accuracy, completeness, or usefulness of any information, apparatus, product, or process disclosed, or represents that its use would not infringe privately owned rights. Reference herein to any specific commercial product, process, or service by trade name, trademark, manufacturer, or otherwise, does not necessarily constitute or imply its endorsement, recommendation, or favoring by the United States Government or the University of California. The views and opinions of authors expressed herein do not necessarily state or reflect those of the United States Government or the University of California, and shall not be used for advertising or product endorsement purposes.

This is a preprint of a paper intended for publication in a journal or proceedings. Since changes may be made before publication, this preprint is made available with the understanding that it will not be cited or reproduced without the permission of the author. 


\title{
Roughness Scaling of Fracture Surfaces in Polycrystalline Materials
}

Eira T. Seppälä, Bryan W. Reed, Mukul Kumar, Roger W. Minich, and Robert E. Rudd Lawrence Livermore National Laboratory, L-415, Livermore, CA 94551, U.S.A.

\begin{abstract}
The roughness scaling of fracture surfaces in two-dimensional grain boundary networks is studied numerically. Grain boundary networks are created using a Metropolis method in order to mimic the triple junction distributions from experiments. Fracture surfaces through these grain boundary networks are predicted using a combinatorial optimization method of maximum flow - minimum cut type. We have preliminary results from system sizes up to $N=22500$ grains suggesting that the roughness scaling of these surfaces follows a random elastic manifold scaling exponent $\zeta=2 / 3$. We propose a strong dependence between the energy needed to create a crack and the special boundary fraction. Also the special boundaries at the crack and elsewhere in the system can be tracked.
\end{abstract}

\section{INTRODUCTION}

Fracture in random media has been of considerable interest in the field of theoretical statistical mechanics for a few decades [1,2], especially in terms of roughness scaling and toughness properties of crack surfaces. However, the statistical mechanics studies of scaling of fracture surfaces in random media have been conducted using simplified, arbitrary models, such as analytical distributions to describe randomness, and regular lattices for the structure of the material. One attempt to study more realistic systems is a recently published study of scaling laws for manifolds in polycrystalline materials, which used a simple model for transgranular and intergranular cracks, but neglected any dynamical effects [3]. Here we propose that polycrystalline materials with varying grain boundary strengths provide a good example of random media $[4,5,6]$, where only intergranular fracture is allowed. The orientation of the grains and boundaries can be essentially random, giving rise to a distribution of strengths among grains.

We study fracture in polycrystalline materials by generating grain boundary networks using a Metropolis method in order to mimic the triple junction distributions for special boundaries from experimental samples. The final fracture surface and its properties are predicted from the structure of the material, in particular from the topology of the grain boundary network and the strength properties of the different boundaries in the network. The method used here for predicting the final fracture surface has been developed in statistical mechanics and shown to be very efficient $[7,8]$. It finds the weakest (minimum energy) path through the material using a combinatorial optimization method [9].

In this paper we especially wish to study how the properties of the predicted fracture surfaces are dependent on the fraction of special boundaries and whether the scaling of roughness $w$ with respect to the system size $L$ follows the random elastic manifold universality class $w \sim L^{\zeta}$, with the characteristic roughness exponent $\zeta=2 / 3$ in two dimensions. 


\section{METHODS}

\section{Generation of grain boundary networks}

The grain boundary networks are created by constructing a Delaunay connectivity network from randomly positioned points. The lines of the networks in fact correspond the dual network of the actual grain boundary network (which can be created using a Voronoi construction and has been done here in order to derive the length of each of the grain boundaries). After the centers of the neighboring grains are connected with the Delaunay network, the types of the grain boundaries are assigned. This is done using a Metropolis algorithm. The mathematics behind the algorithm is presented in an article [10]. In the Metropolis algorithm the temperature (an abstract, dimensionless parameter in this case, not a real physical temperature) controls the fraction of the special grain boundaries: the higher the inverse temperature $\left(\beta=1 / k_{B} T\right)$, the greater the fraction of special boundaries, due to their low energies. Because of the low interfacial energies the special boundaries are also stronger (more fracture resistant) than random boundaries. Each of the networks was started from a random configuration and equilibrated with a number of Monte Carlo steps equal to 1000 times the grain count. Here we treat as special boundaries, the boundaries with $\Sigma=3^{n}, n=0,1,2,3,4$, or $\Sigma 1, \Sigma 3, \Sigma 9, \Sigma 27, \Sigma 81$; and the rest are functionally equivalent random boundaries.

In order to study the strength properties of such networks a theoretical, idealized strength per length function was chosen, $\sigma=(57 / \Sigma)+1$ for special boundaries and $\sigma=1$ for random boundaries, such that $\Sigma=3$ ( $\Sigma 3$ boundary) is 20 times stronger than a random boundary. The $\sigma$-value of each grain boundary is naturally multiplied by its length giving $\sigma_{l}$.

\section{Weakest paths through the networks}

The method to predict the fracture surface is to find the global weakest path through the network [7, 8, 9]. This has been done by creating a flow network from the Delaunay connectivity network between grains. In this flow network each edge of the network has a capacity depending on the strength value $\sigma_{l}$ of the grain boundary. Two extra vertices are introduced: a source and a sink. The source and the sink are at opposite sides of the network mimicking mode I -loading. So called maximum flow - minimum cut algorithms have been proved to give an exact solution for finding the bottleneck of the network where one tries to push as much flow as possible from the source to the sink [11]. This bottleneck is the global lowest energy or weakest path solution to cut the random network in two parts. We have used an efficient version of the network developed by Goldberg [12], and it scales nearly linearly with respect to the number of vertices in the system and solves a problem with $10^{6}$ vertices in one minute of CPU-time on a standard workstation.

\section{RESULTS}

\section{Special boundary fraction and triple junction distributions}

For the purpose of this study, scaling of the roughness of the fracture surfaces, we created grain boundary networks with different sizes, the number of grains varying as $N=225,1000,2500,6400$, 10000 , and 22500. For each of the networks we had $N_{r}$ different random configurations, such that $N_{r}=2500($ for $N=225), 1000(N=1000), 250(N=2500), 100(N=6400), 60(N=10000)$, 

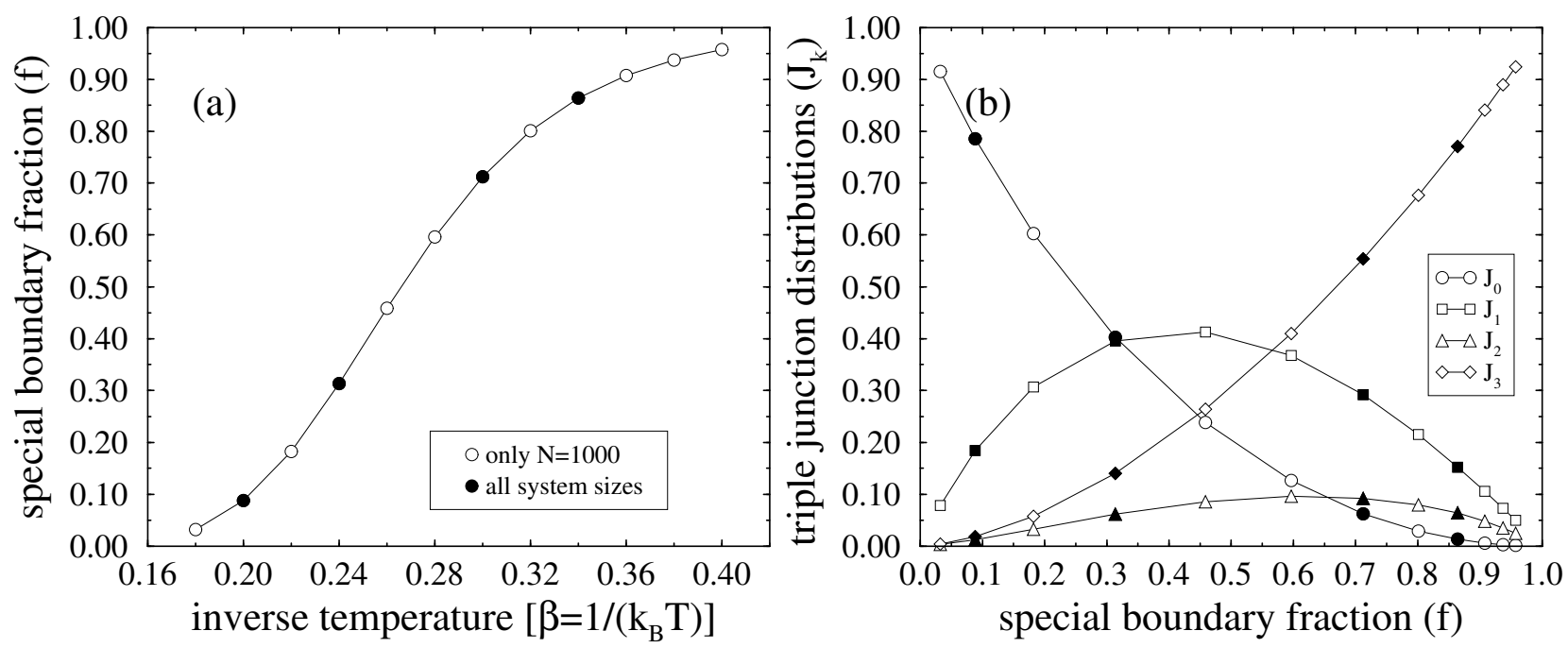

Figure 1. (a) Special boundary fraction $f$ versus inverse temperature $\beta$ and (b) triple junction distributions $J_{k}$ from networks with $N=1000$ grains and $N_{r}=1000$ random configurations at each temperature. Filled symbols represent those $f$ and $\beta$ values for which different grain boundary network sizes were generated. The triple junction distributions differ from naive random, binomial distributions.

and $25(N=22500)$. For each of the networks we used four different inverse temperatures, $\beta=0.20,0.24,0.30$, and 0.34 . The inverse temperatures correspond to four different special boundary fractions $f$, or fraction of the boundaries which are special of all the boundaries in the network, $f=0.09,0.31,0.70,0.85$, see figure 1 .

In order to find the special boundary fraction with respect to the inverse temperature as well as the triple junction distributions, we generated networks with $N=1000\left(N_{r}=1000\right)$ and extra inverse temperatures $\beta=0.18,0.22,0.26,0.28,0.32,0.36$, and 0.38 . In figure 1(a) the relationship between the inverse temperature and the special boundary fraction is shown. Figure 1(b) shows from all the triple junctions in the networks, the $J_{k}$ value where $k$ is the number of the special boundaries in the junction. The triple junction distributions differ from the random configurations (the random result is that $J_{1}$ and $J_{2}$ are symmetric with each other, as are $J_{0}$ and $J_{3}$ ) as well as from the experimental values [5]. In the experiments $\Sigma 1$ boundaries are neglected and, indeed, our algorithm reproduces the correct distributions when the triple junctions with $\Sigma 1$ boundaries are not taken into account. This result will be published elsewhere [13].

\section{Roughness and strength with varying system size}

Figure 2(a) shows an example of a network with $N=22500$ grains $(\beta=0.30, f=0.70)$ and with the weakest path through the system or the predicted fracture surface or, what we call here, the crack. In figure 2(b) we have calculated the fraction of the types of the boundaries everywhere in the system and at the crack for a system with $N=22500$ grains and $\beta=0.34$ or $f=0.85$. The statistics are from $N_{r}=25$ random configurations. As visible from the figure, $\Sigma 1$ and $\Sigma 3$ boundaries are unfavored in the crack. For lower special boundary fractions even larger $\Sigma$ values are unfavored: at $f=0.09$ even the $\Sigma 81$ peak drops, for $f=0.31 \Sigma 27$ is the highest special boundary value for which the fraction drops, and for $f=0.70 \Sigma 9$ drops but $\Sigma 27$ and $\Sigma 81$ increase. 

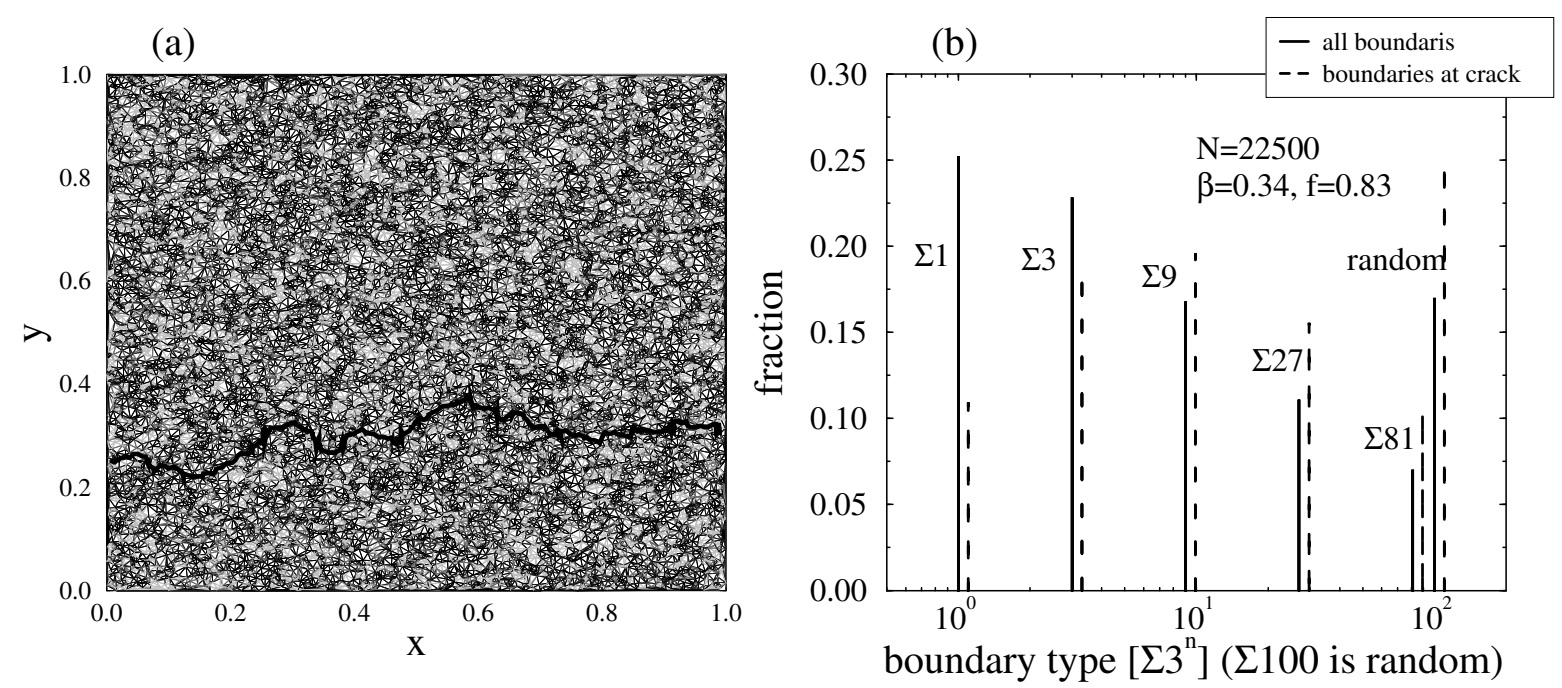

Figure 2. (a) An example of the grain boundary network with $N=22500$ grains and $\beta=0.30$ or $f=0.70$. The darker the line, the stronger the grain boundary. The black thick line is the predicted crack from which the roughness is measured in root-mean-square-fluctuations sense. (b) Special boundaries at the crack and elsewhere in the system with $N=22500$ grains $\left(N_{r}=25\right)$ and $\beta=0.34$ or $f=0.85$. The number of $\Sigma 1$ and $\Sigma 3$ boundaries is smaller at the crack than elsewhere in the system. The population of the other boundary types is increased at the crack.

We have checked that this result is independent of the system size.

The roughness is calculated in the root-mean-square-fluctuations sense, $w=\left\langle(y-\bar{y})^{2}\right\rangle^{1 / 2}$, from the height of the surface as depicted in figure 2(a). Figure 3(a) shows the scaling of the roughness $w$. Although much better statistics are needed and the requisite calculations are underway there is an indication, that it follows the universal random elastic manifold exponent $\zeta=2 / 3$. It is interesting to note that the biggest amplitude $a$ from $w \sim a L^{\zeta}$ is not for the largest $f$ but at an intermediate special boundary fraction.

The energy or the total strength (sum of the strength values $\sigma_{l}$ belonging to the crack) of the crack is plotted in figure 3(b) showing that it scales linearly with is length, which is in line with the random elastic manifold scaling. We use here and throughout the article the term energy to describe the total strength due to the connection of the weakest path algorithm with minimum energy surfaces. Figure 3(b) shows, too, that the energy is strongly dependent on the special boundary fraction.

\section{Roughness and energy with varying special boundary fraction}

We also calculate the roughness and energy with respect to the special boundary fraction for a constant system size $N=1000$ grains. As visible in figure 4(a) the roughness is indeed the highest for the intermediate special boundary fractions and lower for small and large special boundary fractions. This is natural when thinking that it is a measure of randomness, and thus related to the susceptibility of the surface. At low special boundary fractions the crack may propagate nearly straight through the material in order to minimize its elastic line energy. At intermediate $f$ values it needs to wander in order to take advantage of the low energy/strength regions in the system. At high $f$ values the wandering becomes too expensive and again straight cracks are preferred. 

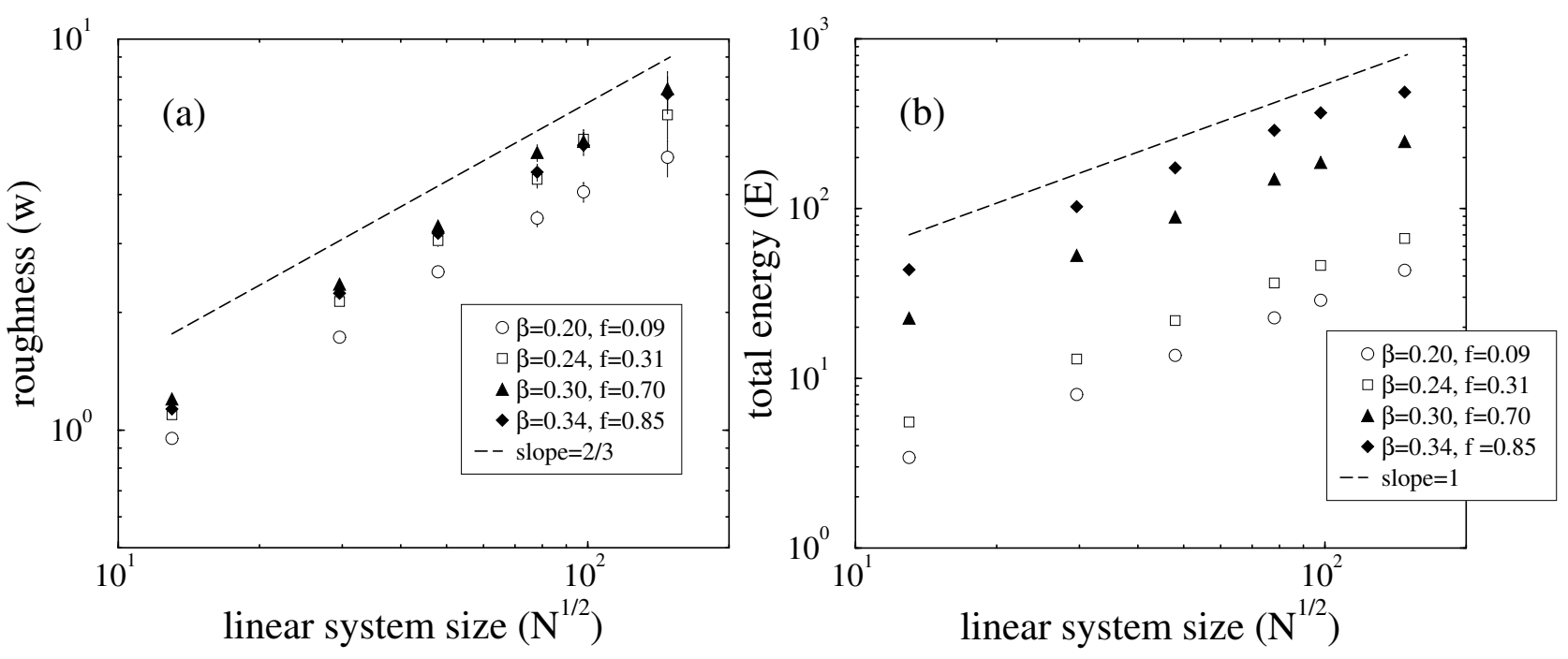

Figure 3. (a) Roughness of the crack and (b) energy (the sum of the strength values of the boundaries at the crack) needed to create it versus linear system size $N^{1 / 2}$ for each of the system size $N=225-22500$ and special boundary fractions $f=0.09,0.31,0.70,0.85$. The roughness follows scaling with an exponent $\zeta=2 / 3$ and the energy is linearly dependent on the linear length of the system.

However, the energy is very strongly dependent on the special boundary fraction, related by the exponential of an exponential function as visible in figure 4(b). Thus all the systems can be broken, but with higher special boundary fractions it costs many times more energy than with lower special boundary fractions.

\section{CONCLUSIONS}

We have studied the fracture of grain boundary networks in two dimensions and have been able to generate efficient algorithms for that purpose. The scaling of the roughness of the fracture surfaces suggests that the fracture paths follow the random elastic manifold scaling with the exponent $\zeta=2 / 3$. Better statistics are needed to confirm this. The elastic manifold scaling implies that the correlations due to the crystallographic constraints are short-range in the sense that they do not change the universality class. We have demonstrated that the population of special boundaries at the crack depends on the overall special boundary fraction, independent of the system size; and that there is very strong dependence between the energy needed to create a crack and the special boundary fraction. It is shown that the roughness is the highest at the intermediate special boundary fractions, too.

In the future we will test different types of algorithms to generate the networks and see if that plays any role. We will also develop more realistic strength functions using molecular dynamics calculations for different types of grain boundaries; including bismuth embrittled boundaries in copper. We aim to compare our results with fracture tests from experimental samples. And although this work was two-dimensional all the computational methods can be extended to and the algorithms already work in three dimensions. 

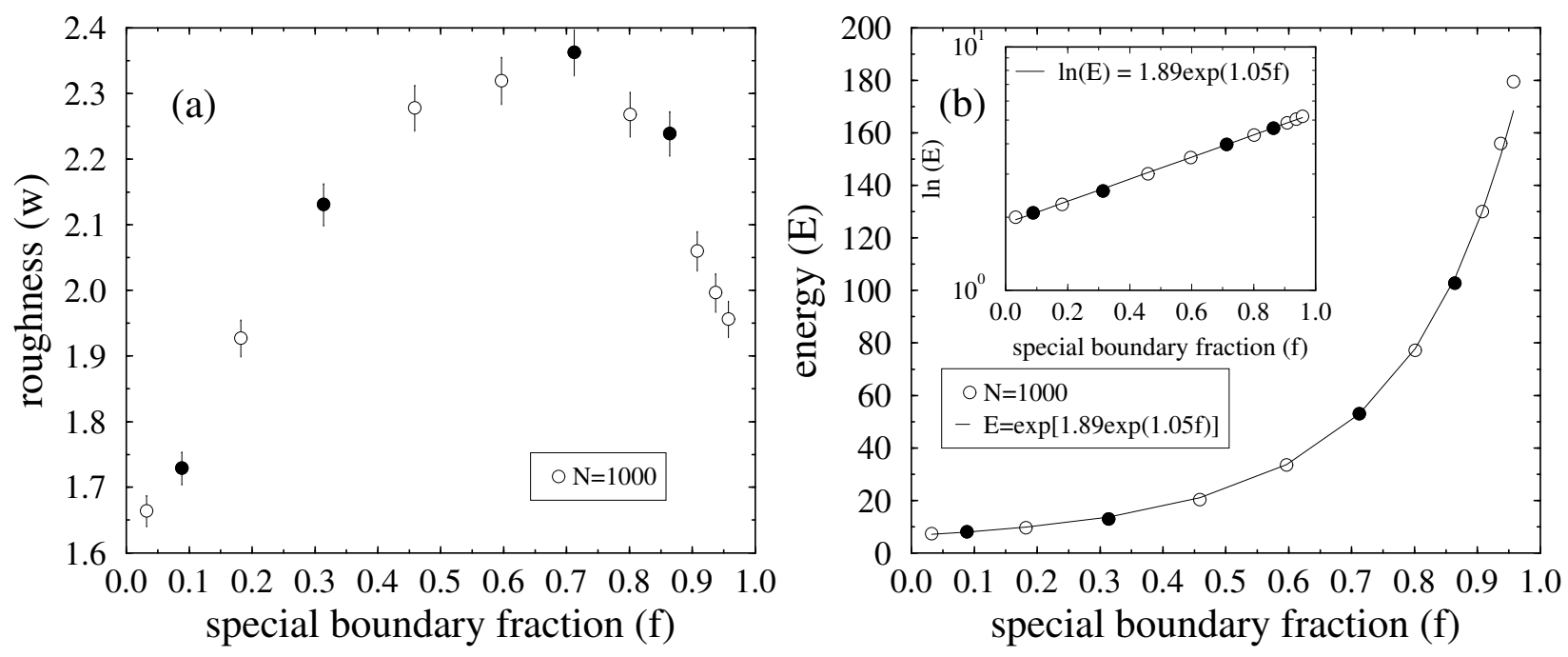

Figure 4. (a) Roughness of the crack and (b) the energy needed to create it versus $f=$ $0.03,0.09,0.18,0.31,0.46,0.60,0.71,0.80,0.86,0.91,0.94$, and 0.96 . The system size is $N=$ 1000 grains and for each $f N_{r}=1000$. The roughness is the highest at intermediate special boundary fractions. The sum of the strength values of the boundaries at the crack depends exponentially to exponential on the special boundary fraction.

\section{ACKNOWLEDGMENTS}

This work was performed under the auspices of the US Dept. of Energy at the University of California/Lawrence Livermore National Laboratory under contract no. W-7405-Eng-48.

\section{REFERENCES}

1. Statistical Models for the Fracture in Disordered Media, edited by H. J. Herrmann and S. Roux, (North-Holland, Amsterdam, 1990).

2. J. Kertész, V. K. Horváth, and F. Weber, Fractals 1, 67 (1993).

3. J. H. Meinke, E. S. McGarry, P. M. Duxbury, and E. A. Holm, Phys. Rev. E 68, 066107 (2003).

4. Randomness in general includes correlated randomness, e.g. that arising under certain geometrical constraints of grain boundaries.

5. R. W. Minich, C. A. Schuh, and M. Kumar, Phys. Rev. B 66, 052101 (2002).

6. C. A. Schuh, M. Kumar, W. E. King, Acta Mater. 51, 687 (2003).

7. V. I. Räisänen, E. T. Seppälä, M. J. Alava, and P. M. Duxbury, Phys. Rev. Lett. 80, 329 (1998).

8. E. T. Seppälä, V. I. Räisänen, and M. J. Alava, Phys. Rev. E 61, 6312 (2000).

9. M. Alava, P. Duxbury, C. Moukarzel, and H. Rieger, Phase Transitions and Critical Phenomena, edited by C. Domb and J. L. Lebowitz (Academic Press, San Diego, 2001), vol 18.

10. B. W. Reed, R. W. Minich, R. E. Rudd, and M. Kumar, Acta Cryst. A 60, 263 (2004).

11. More details of maximum flow - minimum cut algorithms can be found from any basic data structure and algorithm book, see e.g., R. Sedgewick, Algorithms in C (Addison-Wesley, Reading, 1990).

12. A. V. Goldberg and R. E. Tarjan, J. Assoc. Comput. Mach. 35, 921 (1988).

13. E. T. Seppälä, B. W. Reed, R. W. Minich, M. Kumar, and R. E. Rudd (unpublished). 